\title{
The ZZ Ceti star G 185-32: new insight from asteroseismology
}

\author{
D. Pech and G. Vauclair
}

\author{
Université Paul-Sabatier, Observatoire Midi-Pyrénées, CNRS/UMR5572, 14 Av. Edouard Belin, 31400 Toulouse, France \\ e-mail: gerardv@ast.obs-mip.fr \\ Received 18 October 2005 / Accepted 29 January 2006
}

\section{ABSTRACT}

\begin{abstract}
One of the brightest pulsating DA white dwarfs (ZZ Ceti stars), G 185-32, shows 19 periods in its power spectrum, from $71 \mathrm{~s}$ to $651 \mathrm{~s}$. Its short periods are among the shortest ones observed in a ZZ Ceti star. The one at 141.9 s shows an amplitude which does not vary with wavelength, in contrast with what the linear theory predicts for $\ell=1$ or $\ell=2$ non-radial $g$-modes. The mass and $T_{\text {eff }}$ of G 185-32 are well constrained by spectroscopic observations and parallax measurement. The aim of the present paper is to take advantage of this rich observational background to derive the fundamental parameters of the star from asteroseismology. We computed a grid of realistic models for G 185-32 and the periods of their adiabatic $\ell=1$ and $\ell=2$ non-radial $g$-modes. We determined the model which fits best the observations. We identify 14 periods as real modes and determine their $\ell, k$, and $m$ values. We show that the remaining 5 periods are linear combinations. We find that G 185-32 oscillates mostly according to $\ell=2$ modes. We present a new hypothesis to account for the peculiar behaviour of the $141.9 \mathrm{~s}$ mode's amplitude and suggest that it is a true mode interfering with higher $\ell$

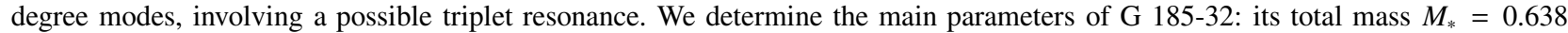
$( \pm 0.007) M_{\odot}$, its hydrogen mass fraction $M_{\mathrm{H}}=1.70( \pm 0.10) \times 10^{-4} M_{*}$, which implies a helium mass fraction of $\approx 10^{-2} M *$, its $T_{\text {eff }}=12280( \pm) 80 \mathrm{~K}$, and estimate its rotation period to be $14.5 \mathrm{~h}$.
\end{abstract}

Key words. stars: fundamental parameters - stars: white dwarfs - stars: oscillations - stars: individul: ZZ Ceti G 185-32

\section{Introduction}

The DA white dwarf G 185-32 (PY Vul or WD1935+279) was discovered to pulsate as a ZZ Ceti star by Mc Graw et al. (1981). It is one of the brightest known ZZ Ceti stars ( $V=12.97 \mathrm{mag})$, and it exhibits many low-amplitude pulsation frequencies, making the star a good candidate for a detailed asteroseismological analysis. Bergeron et al. $(1995,2004)$ determined its full mass and effective temperature of $M_{\star}=0.64( \pm 0.03) M_{\odot}$ and $T_{\text {eff }}=12130( \pm 200) \mathrm{K}$, from high resolution, high signal/noise spectroscopy and from a model atmosphere using the ML2 version of the mixing length theory with $\alpha=0.6$. This $T_{\text {eff }}$ places G 185-32 close to the blue edge of the ZZ Ceti instability strip.

Photometric observations of G 185-32 were obtained during the 1992 Whole Earth Telescope (WET, Nather et al. 1990) campaign XCOV8 (photometry in the visible wavelength range) and by Kepler et al. (2000) with the HST Faint Object Camera (UV and far blue photometry). From these observations, 19 periodicities have been detected between $71 \mathrm{~s}$ and $651 \mathrm{~s}$. With periods as short as $70 \mathrm{~s}, \mathrm{G} 185-32$ is the shortest-period ZZ Ceti pulsator confirmed so far ${ }^{1}$. In addition, time-resolved spectroscopy has been obtained by Thomson et al. (2004) with one Keck telescope.

Castanheira et al. (2004, hereafter CKM) present a preliminary study of G 185-32 based on the WET and HST observations. They tried to discriminate the true pulsation modes from their linear combinations. They also proposed an identification of the spherical degree, $\ell$, for most of the observed periods by using their amplitude variation with wavelength as predicted by linear theory for $\ell=1$ and $\ell=2$ non-radial $g$-modes. The power spectrum of $\mathrm{G} 185-32$ shows one puzzling period at $141.9 \mathrm{~s}$

1 Mukadam et al. (2006) report the discovery of an even shorter period of $48.5 \mathrm{~s}$ in the new ZZ Ceti star WD0939+5609 which needs confirmation. whose amplitude does not increase toward the UV contrarily to what is predicted for $g$-modes pulsation of degree $\ell=1$ or $\ell=2$ (Robinson et al. 1982, 1995; Kepler 1984). As a consequence, they conclude that it should be a false mode, probably the subharmonic of the $70.9 \mathrm{~s}$ period. Accordingly, this suggestion implies that the $70.9 \mathrm{~s}$ is a real mode, so their study relies on the assumption that it is the $\ell=2, k=1$ mode. Thompson et al. (2004) propose a different hypothesis for the peculiar behaviour of the $141.9 \mathrm{~s}$ period. They suggest that it could be an $\ell=4$ mode whose amplitude is predicted as not changing significantly with wavelength.

These interpretations of the observational data do not use any model computations. Such a modelling is the next step in checking whether one model, or set of models, could realistically fit the observed frequencies. It is also useful when trying to recognize true pulsation modes from their linear combinations, since such linear combinations might actually be genuine modes involved in true resonances. In addition, they did not consider the rotational splitting effect and thus did not take the impact of stellar rotation on the observed modes into account. This context strongly motivated us to undertake an independent study following a different approach, preferably based on modelling. This is the next step in deciphering the power spectrum of G 185-32 and trying to derive its fundamental parameters.

In Sect. 2, we summarize the main observational data on G 185-32. In Sect. 3, we discuss our strategy for modelling the star and obtaining a best-fitting model. In Sect. 4 we present a first identification of the degree $\ell$ and order $k$ of the observed modes and discuss the cases of the ambiguous frequencies; for each of them, we find out whether they are a linear combination or a true mode. Section 5 introduces the effect of the rotational splitting that enables us to complete the modes identification with the azimuthal number $m$. In Sect. 6, we discuss our identifications and focus on the case of the mysterious $141.9 \mathrm{~s}$ period 
Table 1. Observed periods in G 185-32.

\begin{tabular}{ll}
\hline \hline $\begin{array}{l}\text { Frequency } \\
(\mu \mathrm{Hz})\end{array}$ & $\begin{array}{l}\text { Period } \\
(\mathrm{s})\end{array}$ \\
\hline 1534.5 & 651.7 \\
$1783.3(!)$ & 560.8 \\
$1860.2(?)$ & 537.6 \\
$2199.9(?)$ & 454.6 \\
2701.2 & 370.2 \\
3317.8 & 301.4 \\
3335.6 & 299.8 \\
$3507.5(?)$ & 285.1 \\
3757.3 & 266.2 \\
3785.2 & 264.2 \\
4635.3 & 215.7 \\
4698.8 & 212.8 \\
$5497.7(?)$ & 181.9 \\
$6736.1(!)$ & 148.5 \\
$7048.8(*)$ & 141.9 \\
7080.4 & 141.2 \\
13714.4 & 72.9 \\
13784.9 & 72.5 \\
$14097.7(!)$ & 70.9 \\
\hline
\end{tabular}

for which we present a new hypothesis. Section 7 summarizes our work.

\section{Observational background}

The available data on G 185-32 are described in CKM. Table 1 lists the 19 significant periods detected in its power spectrum, following their analysis. The question mark indicates periods that were marginally detected, either in the WET or in the HST data sets, or, in the case of the $285.1 \mathrm{~s}$ period, was only detected by Thomson et al. (2004). The exclamation mark indicates frequencies that are implied in a linear combination (or a true resonance), so that their identification as real modes is doubtful. The asterisk outlines the enigmatic $141.9 \mathrm{~s}$ period whose amplitude remains constant with wavelength.

A first examination of Table 1 suggests that the G 185-32 pulsation spectrum seems to exhibit partial multiplets. That is the case for the pairs of periods: $299.8 \mathrm{~s}-301.4 \mathrm{~s} ; 264.2 \mathrm{~s}-266.2 \mathrm{~s}$, and the potential triplet $72.5 \mathrm{~s}-72.9 \mathrm{~s}-70.9 \mathrm{~s}$. Those groups have periods that are close enough to be considered a priori as members of the same multiplet generated by rotational or magnetic splitting. Let us consider the case of the magnetic splitting first. Since the expected frequency shift is proportional to $\mathrm{m}^{2}$, the ratio of the frequency differences between the $m=2$ and the $m=1$ components and the $m=1$ and the $m=0$ components should be 4 , if this triplet corresponds to the $m=0,1,2$ components of an $\ell=2$ mode. The observed ratio is $4.43 \pm 0.18$ from the WET data, where we use the frequency resolution of the WET campaign as an upper limit to the frequency uncertainty. This is close enough to the expected value of 4 to make the assumption of magnetic splitting appealing. However, according to Jones et al. (1989) a magnetic field largely in excess of $10^{5} \mathrm{G}$ would be required in order to reproduce the observed frequency shift of $70 \mu \mathrm{Hz}$ between the presumed $m=0$ and $m=1$ components with periods $72.9 \mathrm{~s}$ and 72.5 s. Since Schmidt \& Smith (1995) reported only an upper limit of $-8.5 \pm 10.5 \mathrm{kG}$ for the magnetic field of $\mathrm{G} \mathrm{185-32}$, one can safely reject the magnetic splitting as being responsible for the observed multiplets. We conclude that the observed multiplets are the components of modes split by rotation.

\section{Modelling strategy}

Pech et al. (2006, hereafter PVD), show in the case of HL Tau 76 how far it was possible to constrain the internal structure of a ZZ Ceti star from asteroseismology. The chosen method relies on building a grid of static models and comparing the observed modes with the synthetic spectrum computed for each of them. The grid of models was built in a 3-dimensional parameter space by varying the total mass, the fractional mass of the hydrogen layer, and the effective temperature. The fractional mass of the helium layer was constrained by the mass of the hydrogen layer, on one hand, and by considerations of the absence of observed carbon in the spectra of ZZ Ceti stars on the other. A similar grid of models was built for the purpose of the present paper in the range of parameters suitable for G 185-32. The strategy used to constrain the model of HL Tau 76 was based on an $\chi^{2}$ test that selects the model that fits best the observations. The strength of this method is to take all the observed modes into account, after removal of the linear combinations. Its efficiency depends on the richness of the observed spectrum. Its weakness, however, is to be sensitive to a preliminary identification of the modes according to their $\ell$ degree. Actually, if the identification of $\ell$ is uncertain for many modes, the $\chi^{2}$ algorithm may link computed and observed modes of different degrees and result in a wrongfitting model. However, if the star exhibits a large enough number of modes, some errors in the preliminary determination of $\ell$ (due to observational uncertainties) do not threaten the reliability of the deduced best-fit model. That was the case for the cool ZZ Ceti star HL Tau 76 (PVD), which exhibits as much as $\approx 40$ independent modes (Dolez et al. 2006). In the case of G 185-32, a smaller amount of periods (19) is available, making the $\chi^{2}$ method risky. It is even more so, since some of them (at least three) are suspected to be false modes involved in linear combinations. That is why we decided to use another strategy.

\subsection{A reference mode}

The first step is to consider a reference mode with a secure identification in the frequency spectrum. It has to be selected among the shortest periods since there are those which constrain the model more unambiguously. There are actually 3 periods to choose from $70.9 \mathrm{~s}, 72.5 \mathrm{~s}$, and $72.9 \mathrm{~s}$. The $72.5 \mathrm{~s}-72.9 \mathrm{~s}$ pairs could be considered at this stage as two possible components of a multiplet. The status of the $70.9 \mathrm{~s}$ is ambiguous, since it could be a true mode or the first harmonics of the $141.9 \mathrm{~s}$ period. For this reason, we did not take it as our reference mode, in contrast to CKM, but chose the shortest period of the pair at $72.5 \mathrm{~s}$.

The visibility of a mode of a given $\ell$ degree decreases rapidly with increasing values of $\ell$ and depends on the inclination angle of the pulsation axis of symmetry on the line of sight (see for instance Dziembowski 1977; Robinson et al. 1982; Brassard et al. 1995). Brassard et al. (1995) have computed the expected visibility of pulsation modes of degree $\ell \leq 5$ for ZZ Ceti stars through various photometric bandpasses. They show that for the typical white light WET photometry, the visibility of $\ell=3$ modes relative to $\ell=1$ modes is reduced by a factor $6.4 \times 10^{-2}$ by taking into account the geometrical cancellation effect resulting from the integration on the stellar disk of the Legendre polynomial associated to the spherical harmonics and assuming that all the modes of various $\ell$ values have the same intrinsic amplitudes. The $\ell=4$ modes' visibility is reduced even more by a factor $3.95 \times 10^{-2}$. As a result it is generally admitted that modes with degrees $\ell \geq 3$ are unlikely to be observed. Thus, the expected value for the degree of the modes corresponding to the periods 
at $72.9 \mathrm{~s}, 72.5 \mathrm{~s}$, or $70.9 \mathrm{~s}$ should be either $\ell=1$ or $\ell=2$. Identifying these periods either as $\ell=1, k \geq 1$ or as $\ell=2, k \geq 2$ would imply a mass of G 185-32 close to the Chandrashekar limit, which strongly disagrees with the mass of $0.64 \pm 0.03 M_{\odot}$ deduced from spectroscopy (Bergeron et al. 1995, 2004). As a consequence, our reference period of $72.5 \mathrm{~s}$ is necessarily the $\ell=2, k=1$ mode. Modelling will confirm this assertion later. We checked whether the $70.9 \mathrm{~s}$ could, however, be an $\ell=3$ mode. Note that it would imply that it should have an intrinsic amplitude at the stellar surface that is at least 15 times larger than the $\ell=1$ modes in order to exhibit a comparable, observed amplitude. We think that this is quite unlikely, but as the intrinsic amplitudes at the stellar surface are unknown, we cannot formally disprove such a possibility. We extended our grid of models to find what should the stellar mass be, in order to have its $\ell=3, k=1$, or $k=2$ mode with a 70.9 s period. We found $0.5 M_{\odot}$ for the case $k=1$ and a mass in excess of $0.9 M_{\odot}$ in the case $k=2$. Both masses are far outside the range of mass allowed by spectroscopy. We conclude that the $70.9 \mathrm{~s}$ is not an $\ell=3$ mode on an asteroseismology basis.

In their paper, CKM also conclude that the shortest period observed in G 185-32 has to be the $\ell=2, k=1$ mode and identified this mode as the $70.9 \mathrm{~s}$ period. In the next sections, we alternatively explore the possibility of accounting for the period distribution observed in G 185-32 by identifying the $72.5 \mathrm{~s}$ with this mode.

\subsection{Determining potential solutions in the $\log q(H)$ vs. $M_{\star}$ plane}

Once the reference mode is selected, the goal was to search for potential solutions in the $\log q(H)=\log M(H) / M_{\star}$ vs. $M_{\star}$ plane, where $M(H)$ is the mass of the hydrogen outer layers. Our method consists in determining a series of couples $\left(\log q(H), M_{\star}\right)$ for which the resulting model presents the $\ell=2$, $k=1$ mode with a period of $72.5 \mathrm{~s}( \pm 0.7 \mathrm{~s})$, i.e. within an allowed $1 \%$ uncertainty. Our model grid scanned the whole range of total mass allowed by spectroscopy (i.e. from $0.61 M_{\odot}$ up to $0.67 M_{\odot}$ ). Then, the hydrogen mass fraction for each model was adjusted so as to obtain the right period for the $\ell=2, k=1$ mode.

Our model grid is built as described in PVD. They are static models with a pure, degenerate carbon core. Convection in the outer $\mathrm{H} / \mathrm{He}$ layers is computed according to the ML2 version of the mixing length theory with $\alpha=0.6$. The remaining free parameters were kept constant because they do not influence the fundamental $(\ell=2, k=1)$ mode enough to alter its period noticeably. Brassard et al. (1992) showed that the periods of pulsation are almost insensitive to the helium mass fraction. Thus this parameter was kept constant at $q(\mathrm{He})=10^{-2}$. The input parameters for the model calculations are the total mass of the white dwarf, the hydrogen and helium fractional masses, and the luminosity. The other global parameters are derived from the model calculated, i.e. the $T_{\text {eff }}$ and the radius. The model with the appropriate value of $T_{\text {eff }}$ is obtained by iterating on the value of the luminosity.

We checked the impact of changing the effective temperature of the models on the period of the reference mode. To do this, we built up 8 models with $M_{\star}$ close to the mass of G 185-32 as deduced from spectroscopy. These models have $M_{\star}=0.62 M_{\odot}, q(\mathrm{H})=10^{-4}$, and $q(\mathrm{He})=10^{-2}$. Their $T_{\text {eff }}$ varies within the spectroscopic range (from $11930 \mathrm{~K}$ to $12330 \mathrm{~K}$ ). For each model, the adiabatic $\ell=2, k=1$ and $\ell=2, k=10$ modes were computed. Table 2 lists the periods of these modes. While
Table 2. Impact of $T_{\text {eff }}$ variation on the $\ell=2, k=1$ and $\ell=2, k=10$ modes.

\begin{tabular}{ccc}
\hline \hline $\begin{array}{c}T_{\text {eff }} \\
(\mathrm{K})\end{array}$ & $\begin{array}{c}\ell=2, k=1 \\
\text { Period }(\mathrm{s})\end{array}$ & $\begin{array}{c}\ell=2, k=10 \\
\text { Period }(\mathrm{s})\end{array}$ \\
\hline 11950 & 79.9 & 379.3 \\
12020 & 79.9 & 378.8 \\
12080 & 79.8 & 376.9 \\
12150 & 79.8 & 373.2 \\
12210 & 79.8 & 370.8 \\
12270 & 79.8 & 367.3 \\
12320 & 79.7 & 365.4 \\
\hline
\end{tabular}

the period of the $\ell=2, k=10$ mode increases by $14 \mathrm{~s}$ when $T_{\text {eff }}$ varies within this range, the period of the $\ell=2, k=1$ mode increases by only $0.2 \mathrm{~s}$. We must emphasize here that since our models are static models, it is not possible to infer the rate of change of the period. To calculate such a rate of change would require a fully evolutionary sequence. The goal of these additional model calculations is to evaluate the sensitivity of the periods of the $\ell=2, k=1$, and $k=10$ modes to the location of the star within the error box in the H-R diagram. Accordingly, the period variation of $0.2 \mathrm{~s}$ that we find for the $\ell=2, k=1$ mode for a variation of $T_{\text {eff }}$ of $370 \mathrm{~K}$ is not a measure of the rate of change for the period expected for a decrease in $370 \mathrm{~K}$ along a cooling sequence. Checking that the period of the reference mode does not change much in the allowed range of $T_{\text {eff }}$ is important since the validity of the possible solutions in the $\log q(H)$ vs. $M_{\star}$ plane strongly relies on the quasi-constancy of that period. Table 2 guarantees that one may consider $T_{\text {eff }}$ as a degenerate parameter in a first step.

The spectroscopic range for $M_{\star}$ was covered with a step of $0.005 M_{\odot}$ from $0.610 M_{\odot}$ to $0.670 M_{\odot}$, and $q(\mathrm{H})$ was fit so each model kept the fundamental mode with a period of $72.5 \mathrm{~s}( \pm 1 \%)$. Then $T_{\text {eff }}$ was fixed at $12130 \mathrm{~K}$ and $q(\mathrm{He})$ at $10^{-2}$.

Then we tried a rough identification of the modes observed in the $\mathrm{G}$ 185-32 spectrum from the adiabatic spectra calculated for each model. Table 3 gives the results: $M_{\star}, \log q(\mathrm{H})$, the number of correctly identified modes, and the respective $\sigma$ for each of the 13 models. Table 3 shows that the model with $M_{\star}=0.640 M_{\odot}$ and $q(\mathrm{H})=1.62 \times 10^{-4}$ has a spectrum that fits the observed modes with the best accuracy (lowest deviation). It is also one of those that identify the larger number of observed modes. Consequently, the mass of G 185-32 and its hydrogen mass fraction should be close to this couple of values. The second step of the process is now to perform a fine determination of these two parameters around this pair of values.

\subsection{Fine determination of $M_{\star}, q(H)$, and $T_{\text {eff }}$}

The models in the second sharper grid have masses around $0.640 M_{\odot}$, with a variation step of $0.002 M_{\odot}$ between two consecutive models. The $\mathrm{H}$ mass fraction is adjusted accordingly to keep the period of the reference mode constant. The $T_{\text {eff }}$ is maintained at $12130 \mathrm{~K}$ for all the models. The best fit with the observed power spectrum of $G$ 185-32 is found for the couple $M_{\star}=0.638( \pm 0.007) M_{\odot}$ and $q(\mathrm{H})=1.70$ $( \pm 0.10) \times 10^{-4}$. The uncertainty on $M_{\star}$ is an estimate of how necessary it is to change the mass from the best-fit value $\left(0.638 M_{\odot}\right)$ to increase the deviation $\sigma$ of the resulting model by $10 \%$. The uncertainty on $q(\mathrm{H})$ corresponds to the minimum variation from the best-fit value $\left(1.70 \times 10^{-4} M_{\star}\right)$ necessary to exceed the $1 \%$ tolerance allowed on the reference period. For a best-fit model with $M_{\star}=0.638 M_{\odot}$, it is the 
Table 3. Potential solutions in the $\log q(\mathrm{H})$ vs. $M_{\star}$ plane derived from the reference mode with $T_{\text {eff }}$ and $q(\mathrm{He})$ constant.

\begin{tabular}{cccc}
\hline $\begin{array}{c}\text { Stellar mass } \\
M_{\star} / M_{\odot}\end{array}$ & $\begin{array}{c}\text { H mass fraction } \\
\log q(\mathrm{H})\end{array}$ & $\begin{array}{c}\text { Identified } \\
\text { modes }\end{array}$ & $\begin{array}{c}\sigma \\
(\mathrm{s})\end{array}$ \\
\hline 0.610 & -3.62 & 10 & 4.8 \\
0.615 & -3.66 & 11 & 4.5 \\
0.620 & -3.74 & 11 & 4.2 \\
0.625 & -3.72 & 12 & 3.9 \\
0.630 & -3.74 & 13 & 3.4 \\
0.635 & -3.76 & 13 & 3.0 \\
0.640 & -3.79 & 13 & 2.8 \\
0.645 & -3.82 & 13 & 3.1 \\
0.650 & -3.86 & 13 & 3.3 \\
0.655 & -3.88 & 12 & 3.6 \\
0.660 & -3.91 & 12 & 3.8 \\
0.665 & -3.95 & 12 & 3.8 \\
0.670 & -3.96 & 10 & 3.7 \\
\hline
\end{tabular}

Table 4. Structural parameters for the best fitting model.

\begin{tabular}{cc}
\hline \hline Stellar mass & $M_{\star}=0.638( \pm 0.007) M_{\odot}$ \\
Temperature & $T_{\text {eff }}=12280( \pm 80) \mathrm{K}$ \\
H-layer mass & $M(\mathrm{H})=1.70( \pm 0.10) \times 10^{-4} M_{\star}$ \\
He-layer mass & $M(\mathrm{He})=10^{-2} M_{\star}$ \\
Luminosity & $L=0.00461 L_{\odot}$ \\
Stellar radius & $R=0.01531 R_{\odot}$ \\
\hline
\end{tabular}

Table 5. $\ell=1$ modes for the best fitting model.

\begin{tabular}{cccc}
\hline \hline$k$ & Period (s) & $k$ & Period (s) \\
\hline 1 & 124.6 & 7 & 461.5 \\
2 & 244.0 & 8 & 505.1 \\
3 & 281.2 & 9 & 533.7 \\
4 & 322.0 & 10 & 589.8 \\
5 & 364.3 & 11 & 637.5 \\
6 & 397.0 & 12 & 680.2 \\
\hline
\end{tabular}

variation of $q(\mathrm{H})$ that pushes the period of the $\ell=2, k=1$ mode outside the range $71.8 \mathrm{~s}-73.2 \mathrm{~s}$.

The value derived for $q(\mathrm{H})$ confirms that the initial choice for the He mass fraction was optimal since such a thick hydrogen envelope implies that $q_{\mathrm{He}}$ must be close to $10^{-2} M_{\star}$. The last free parameter to constrain is $T_{\text {eff }}$. We calculated the power spectra for a series of models with $M_{\star}=0.638 M_{\odot}$ and $q(\mathrm{H})=1.70 \times 10^{-4}$, varying their $T_{\text {eff }}$ within the spectroscopic interval $(11920 \mathrm{~K}-12320 \mathrm{~K})$ by $40 \mathrm{~K}$ steps. For each model, we evaluated its deviation $\sigma$. The impact of changing $T_{\text {eff }}$ on the pulsation periods is very weak. However, the value of $\sigma$ indicates that the model with $T_{\text {eff }}=12280 \mathrm{~K}$ gives the best fit. A variation of $80 \mathrm{~K}$ around this value does not change the quality of the fit significantly. We conclude that the most probable effective temperature is $T_{\text {eff }}=12280( \pm 80) \mathrm{K}$. This best-fit model gives $\sigma=2.0 \mathrm{~s}$, with an average relative error of $0.63 \%$ on the periods. The details of this fit are discussed in the next section.

The model fit procedure described in Sect. 3.2 results in the determination of the main structural parameters of G 185-32: its total mass $M_{\star}$, its $\mathrm{H}$ and He mass fractions, its luminosity, its $T_{\text {eff }}$, and its radius. These parameters are summarized in Table 4.
Table 6. $\ell=2$ modes for the best fitting model.

\begin{tabular}{cccc}
\hline \hline$k$ & Period (s) & $k$ & Period (s) \\
\hline 1 & 72.0 & 13 & 431.5 \\
2 & 142.1 & 14 & 455.2 \\
3 & 163.4 & 15 & 474.2 \\
4 & 194.3 & 16 & 508.8 \\
5 & 224.2 & 17 & 542.1 \\
6 & 240.1 & 18 & 559.6 \\
7 & 269.3 & 19 & 587.6 \\
8 & 300.7 & 20 & 605.0 \\
9 & 326.4 & 21 & 633.8 \\
10 & 346.2 & 22 & 672.3 \\
11 & 374.2 & 23 & 698.6 \\
12 & 397.0 & 24 & 712.2 \\
\hline
\end{tabular}

\section{Preliminary study of the observed modes of $\mathrm{G}$ 185-32}

\subsection{Preliminary identification of the modes}

During the process described in the previous section, we computed the adiabatic $\ell=1$ and $\ell=2$ pulsation modes for the best-fit model on a period interval encompassing the range of periods observed in $\mathrm{G} 185-32$. The periods of those modes are listed in Table 5 for the $\ell=1$ modes and in Table 6 for the $\ell=2$ ones. This enables us to perform a preliminary identification of the observed pulsations. It also allows us to distinguish for ambiguous cases whether the observed periods are true modes or linear combinations. At this stage, we do not yet consider the impact of the stellar rotation on the pulsation spectrum, so that those periods are for the $m=0$ component of each mode.

Table 7 shows the comparison between the observed periods and the periods calculated in the best-fit model. The average deviation for this preliminary identification is $\sigma=2.0 \mathrm{~s}$ and the average relative difference is $|\delta P / P|=0.63 \%$. These two indicators reveal a satisfactory fit between observed and computed spectra. This fit will be refined later and completed when the azimuthal number $m$ is taken into account.

\subsection{Linear combinations or true resonances?}

Using the preliminary identification of the modes given in Table 7 , one can check which frequencies must correspond to real modes and which ones are linear combinations of parent modes. Some of the observed periods cannot be recovered by the synthetic spectrum of the best fit model, which implies that they should be false modes.

Let us consider first the mysterious $141.9 \mathrm{~s}$ period. The model spectrum has its $\ell=2, k=2$ mode at a period of $142.1 \mathrm{~s}$. This suggests that the pair $141.2 \mathrm{~s}-141.9 \mathrm{~s}$ is part of the quintuplet generated by the $\ell=2, k=2$ mode split by rotation. Accordingly, the $141.9 \mathrm{~s}$ is a true mode and the $70.9 \mathrm{~s}$ should consequently be its harmonic, thus a false mode $\left(f_{70.9 \mathrm{~s}}=2 \times f_{141.9 \mathrm{~s}}\right.$ with $|\Delta f|=\left|f_{\text {obs }}-f_{\text {comp }}\right|=0.05 \mu \mathrm{Hz}$, where $f_{\text {obs }}$ is the observed frequency and $f_{\text {comp }}$ the computed frequency).

The $148.5 \mathrm{~s}$ period does not correspond to any computed mode. This confirms its identification as a linear combination satisfying the relation: $f_{148.5 \mathrm{~s}}=f_{72.5 \mathrm{~s}}-f_{141.9 \mathrm{~s}}$, as suggested by CKM.

The $181.9 \mathrm{~s}$ period is marginally detected only in the HST data set where it appears with a low $\mathrm{S} / \mathrm{N}$ ratio (about 3.6), close to the detection limit that CKM fixed at $3.3\langle A\rangle$, where $\langle A\rangle$ is 
the average noise level. Had they chosen a more conservative detection limit ( $4\langle A\rangle$ for instance), this pulsation would have remained unnoticed and regarded as noise. As a consequence, we are not surprised that the synthetic spectrum derived from our best-fit model fails to reproduce this period. We conclude that the $181.9 \mathrm{~s}$ is not a true pulsation mode.

The period at $651.7 \mathrm{~s}$ might be a linear combination, since $f_{651.7 \mathrm{~s}}=f_{301.4 \mathrm{~s}}-f_{560.8 \mathrm{~s}}$ with $|\Delta f|=0.00 \mu \mathrm{Hz}$. CKM also note that this frequency might be involved in a linear combination, which they expressed as $f_{560.8 \mathrm{~s}}=f_{301.4 \mathrm{~s}}-f_{651.7 \mathrm{~s}}$. The $651.7 \mathrm{~s}$ period is not exactly predicted by the model (Table 7). The calculated $(m=0)$ modes with the closest periods are the $\ell=1$, $k=11$ at $637.5 \mathrm{~s}$ and the $\ell=2, k=22$ at $672.3 \mathrm{~s}$. However, when the rotational splitting is taken into account in the matching process, as discussed in the next section, this period can be better identified with one mode of the synthetic spectrum of the model. In addition, if we look at the amplitudes of these 3 pulsations in the 3 data sets available (WET, HST UV and HST far blue), we note that the $560.7 \mathrm{~s}$ is undetected in the WET data. At first glance, that would suggest that there are 2 real modes: the $301.4 \mathrm{~s}$ and the $651.7 \mathrm{~s}$ periods. Then, when the HST data alone are examined, the three periodicities are detected and the $651.7 \mathrm{~s}$ period may be seen as a linear combination since it has the smallest amplitude. The compatibility between these two different conclusions is therefore ensured if we consider that the three modes are real. This also agrees with our analysis since the three periods are correctly reproduced by our model. Accordingly, we conclude that these three modes are an illustration of a true resonance. Furthermore, the $301.4 \mathrm{~s}$ mode must be a component of a multiplet with the $299.8 \mathrm{~s}$ mode.

The $212.8 \mathrm{~s}$ and $215.7 \mathrm{~s}$ periodicities also deserve close examination. Table 7 shows that these periods cannot be well reproduced with any real mode of the best-fit model. The period of the closest mode $(\ell=2, k=5, m=0)$ is $11.4 \mathrm{~s}$ and $8.5 \mathrm{~s}$ away, respectively. This suggests that those periods should be false modes from a linear combination. However, none of the observed pulsations, properly recognized as true modes in the best-fit model, are able to reproduce these periods by any of their linear combinations. A close inspection of Tables 5 and 6 reveals a perfect coincidence between the $\ell=1, k=6$ and the $\ell=2, k=12$ modes, which pulsate at the same period of $397.0 \mathrm{~s}$. This similarity in periods is within a probable uncertainty close to $0.5 \mathrm{~s}$ due to modelling internal errors. These two modes should interfere. Since this period is not detected in the data, it means that the beating period might be quite long and that the two modes must have been in a situation of destructive interference during the observational campaigns. However, this double $397.0 \mathrm{~s}$ period, although not seen during the observations, might be present in the star as truly excited modes able to generate a linear combination with another parent mode. We note that $f_{212.8 \mathrm{~s}}=f_{454.6 \mathrm{~s}}+f_{\sim 397.0 \mathrm{~s}}$ with $|\Delta f| \sim 20.0 \mu \mathrm{Hz}$ and that $f_{215.7 \mathrm{~s}}=f_{454.6 \mathrm{~s}}+f_{\sim 397.0 \mathrm{~s}}$ with $|\Delta f| \sim 83.0 \mu \mathrm{Hz}$. Note also that these frequency mismatches might be reduced if the proposed linear combinations involve one of the components of the multiplets induced by rotational splitting on the modes at $397.0 \mathrm{~s}$, rather than their $m=0$ component. This will be discussed in the next section. The impossibility of properly matching the $212.8 \mathrm{~s}$ and $215.7 \mathrm{~s}$ periods with any of the computed modes and the possibility of accounting for them as linear combinations involving a beat phenomenon suggests that they are false modes. Our interpretation could be tested by further observations since we suggest that there should be two modes of different degree at the same period of $397.0 \mathrm{~s}$ that were not observed, because they were in destructive interference. There should be circumstances where they are in constructive interference and where that period could be detected.

This preliminary modes identification allows us to conclude that the $70.9 \mathrm{~s}, 148.5 \mathrm{~s}, 181.9 \mathrm{~s}, 212.8 \mathrm{~s}$, and $215.7 \mathrm{~s}$ periods do not correspond to real modes.

\section{The stellar rotation rate and its signature on the pulsation modes}

\subsection{Evaluation of the rotational splitting effect on the observed modes}

A few modes in the frequency spectrum of G 185-32 can be used to estimate the rotational splitting and derive the rotation rate of the star. Some pairs of modes have periods that are close enough to imply that they must be components of a multiplet induced by stellar rotation. As can be seen from Tables 5 and 6 , the adiabatic calculations for the $\ell=1$ and $\ell=2$ modes show that at the periods of those pairs of modes there is no coincidence of periods between modes with different spherical degrees. Thus the existence of these pairs of modes can only be interpreted as the result of rotational splitting, where only two components of the multiplets are seen. From the previous analysis, we identify as such multiplet components the following pairs:

$\mathrm{A}(299.8 \mathrm{~s}, 301.4 \mathrm{~s}), \mathrm{B}(264.2 \mathrm{~s}, 266.2 \mathrm{~s}), \mathrm{C}(141.2 \mathrm{~s}, 141.9 \mathrm{~s})$ and $\mathrm{D}(72.5 \mathrm{~s}, 72.9 \mathrm{~s})$.

All are identified as $\ell=2$ modes. These modes have short periods, which implies that the rotational splitting cannot be determined very precisely. In the following, we consider that the rotation affects all the modes uniformly, whatever their period range. This simplifying hypothesis is justified since a differential rotation, if present, has a weak impact on the pulsation periods. In this case and after assuming slow rotation, the pulsation frequencies in the rotating case, $\sigma_{k, \ell, m}$, are related to the frequencies in the non-rotating case $\left(\sigma_{k, \ell}\right)$ by $\sigma_{k, \ell, m}=\sigma_{k, \ell}+m \times\left(1-C_{k, \ell}\right) \times \Omega$, where $C_{k, \ell}=1 / \ell(\ell+1)$ in the asymptotic limit (Brickhill, 1975) and $\Omega$ is the angular rotation velocity.

To estimate the rotational splitting, one considers the frequency shift between the components of the four pairs of $\ell=2$ modes identified above. We get $\Delta f_{\mathrm{A}}=17.8 \mu \mathrm{Hz}$ for the pair $\mathrm{A}, \Delta f_{\mathrm{B}}=27.9 \mu \mathrm{Hz}$ for $\mathrm{B}, \Delta f_{\mathrm{C}}=31.6 \mu \mathrm{Hz}$ for $\mathrm{C}$, and $\Delta f_{\mathrm{D}}=70.5 \mu \mathrm{Hz}$ for $\mathrm{D}$. Within the asymptotic regime, one should have $\Delta f_{\mathrm{A}}=|\Delta m|_{\mathrm{A}} \times \delta f_{\ell=2}, \Delta f_{\mathrm{B}}=|\Delta m|_{\mathrm{B}} \times \delta f_{\ell=2}$ and $\Delta f_{\mathrm{C}}=|\Delta m|_{\mathrm{C}} \times \delta f_{\ell=2}$, where $\delta f_{\ell=2}$ is the predicted frequency shift for two consecutive components of a quintuplet in the asymptotic regime and $|\Delta m|_{i}$ is the absolute difference between the azimuthal number of the components in each pair $i$.

According to theory, the asymptotic regime may not be verified for the pair $\mathrm{C}(k=2)$. However, the asymptotic relation $\Delta f_{C}=|\Delta m|_{C} \times \delta f_{\ell=2}$ appears surprisingly well satisfied, as seen below. But the asymptotic regime is not verified for the pair D, as expected for a $k=1$ mode.

As $\Delta f_{\mathrm{B}} \sim \Delta f_{\mathrm{C}} \sim 2 \times \Delta f_{\mathrm{A}}$, the possible solutions for $|\Delta m|$ are:

- either $\left|\Delta m_{\mathrm{A}}\right|=1$ and $\left|\Delta m_{\mathrm{B}}\right|=\left|\Delta m_{\mathrm{C}}\right|=2$, which involves a frequency shift for the $\ell=2$ modes: $\delta f_{\ell=2} \sim 16 \mu \mathrm{Hz}$;

- or $\left|\Delta m_{\mathrm{A}}\right|=2$ and $\left|\Delta m_{\mathrm{B}}\right|=\left|\Delta m_{\mathrm{C}}\right|=4$, which implies a frequency shift for the $\ell=2$ modes: $\delta f_{\ell=2} \sim 8 \mu \mathrm{Hz}$.

The pair D allows to remove this indetermination because $\left|\Delta m_{D}\right|$ should be of the order of $\Delta f_{\mathrm{D}} / \delta f_{\ell=2}$. A value for $\delta f_{\ell=2}$ close to $8 \mu \mathrm{Hz}$ can be rejected since it would imply $\left|\Delta m_{\mathrm{D}}\right| \sim 9$, i.e. $\left|\Delta m_{\mathrm{D}}\right| \gg 4$, which contradicts the $\ell=2$ identification. With $\delta f_{\ell=2} \sim 16 \mu \mathrm{Hz}$, then $\left|\Delta m_{\mathrm{D} \text { th }}\right|=4.4\left(\left|\Delta m_{\mathrm{D} \text { th }}\right|\right.$ stands for the 
Table 7. Preliminary identification of the modes observed in G 185-32. The first column gives the observed periods, the second one the spherical degree $(\ell)$ of the mode, the third its radial order $(k)$, the fourth gives the value of the corresponding period computed from the best-fit model, the fifth gives the absolute difference between the two periods, and the sixth their relative difference.

\begin{tabular}{cccccc}
\hline \hline $\begin{array}{c}\text { Observed } \\
\text { period }\end{array}$ & $\begin{array}{c}\text { Degree } \\
\ell\end{array}$ & $\begin{array}{c}\text { Order } \\
k\end{array}$ & $\begin{array}{c}\text { Computed } \\
\text { period }\end{array}$ & $\begin{array}{c}|\delta P| \\
(\mathrm{s})\end{array}$ & $\begin{array}{c}|\delta P / P| \\
(\%)\end{array}$ \\
\hline $70.9 \mathrm{~s}$ & $?$ & $?$ & $?$ & $?$ & $?$ \\
$72.5 \mathrm{~s}$ & 2 & 1 & $72.0 \mathrm{~s}$ & $0.5 \mathrm{~s}$ & 0.7 \\
$72.9 \mathrm{~s}$ & 2 & 1 & & & \\
$141.2 \mathrm{~s}$ & 2 & 2 & & & \\
$141.9 \mathrm{~s}$ & 2 & 2 & $142.1 \mathrm{~s}$ & $0.2 \mathrm{~s}$ & 0.1 \\
$148.5 \mathrm{~s}$ & $?$ & $?$ & $?$ & $?$ & $?$ \\
$181.9 \mathrm{~s}$ & $?$ & $?$ & $?$ & $?$ & $?$ \\
$212.8 \mathrm{~s}$ & $?$ & $?$ & $?$ & $?$ & $?$ \\
$215.7 \mathrm{~s}$ & $?$ & $?$ & $?$ & $?$ & $?$ \\
$264.2 \mathrm{~s}$ & 2 & 7 & & & \\
$266.2 \mathrm{~s}$ & 2 & 7 & $269.3 \mathrm{~s}$ & $3.1 \mathrm{~s}$ & 1.2 \\
$285.1 \mathrm{~s}$ & 1 & 3 & $281.2 \mathrm{~s}$ & $3.9 \mathrm{~s}$ & 1.4 \\
$299.8 \mathrm{~s}$ & 2 & 8 & & & \\
$301.4 \mathrm{~s}$ & 2 & 8 & $300.7 \mathrm{~s}$ & $0.7 \mathrm{~s}$ & 0.2 \\
$370.2 \mathrm{~s}$ & 2 & 11 & $374.2 \mathrm{~s}$ & $4.0 \mathrm{~s}$ & 1.1 \\
$454.6 \mathrm{~s}$ & 2 & 14 & $455.2 \mathrm{~s}$ & $0.6 \mathrm{~s}$ & 0.1 \\
$537.6 \mathrm{~s}$ & 1 & 9 & $533.7 \mathrm{~s}$ & $3.9 \mathrm{~s}$ & 0.7 \\
$560.8 \mathrm{~s}$ & 2 & 18 & $559.6 \mathrm{~s}$ & $1.2 \mathrm{~s}$ & 0.2 \\
$651.7 \mathrm{~s}$ & $?$ & $?$ & $?$ & $?$ & $?$ \\
\hline & & & & & \\
\hline
\end{tabular}

expected value of $\left|\Delta m_{\mathrm{D}}\right|$ in the asymptotic limit). As this approximation is not valid for this doublet (with $k=1$ ), one must have: $C_{1,2 \text { real }} \leq C_{1,2 \text { th }}$ and thus $\left|\Delta m_{\mathrm{D} \text { real }}\right| \leq\left|\Delta m_{\mathrm{D} \text { th }}\right|$. One deduces that $\left|\Delta m_{\text {D real }}\right|=4$.

As a conclusion, we find that there is only one possible value of the frequency shift for the $\ell=2$ modes that satisfies the frequency differences among the four partial quintuplets observed in G 185-32: $\delta f_{\ell=2} \sim 16 \mu \mathrm{Hz}$.

\subsection{Stellar rotation rate and further remarks}

We take advantage of our evaluation of the frequency shift for the $\ell=2$ modes to derive the frequency shift for the $\ell=1$ modes since $\delta f_{\ell=1}=0.6 \times \delta f_{\ell=2}$, within the asymptotic limit. Accordingly, $\delta f_{\ell=1} \sim 10 \mu \mathrm{Hz}$. This value will be useful to complete the identification, with their azimuthal number $m$, of the observed modes that our model identify as $\ell=1$ modes.

\subsubsection{The rotation rate}

The value derived for $\delta f_{\ell=2}$ implies an average rotation period close to $14.5 \mathrm{~h}$. This rotation rate is similar for G 226-29 (about $9 \mathrm{~h}$ ), for which $\delta f_{\ell=1}=16.15 \mu \mathrm{Hz}$ (Kepler et al. 1995). Dolez et al. (2006) derived a value of $\delta f_{\ell=2}=4.23 \mu \mathrm{Hz}$ for HL Tau 76. Therefore G185-32 rotates about 4 times faster than HL Tau 76.

\subsubsection{The $70.9 \mathrm{~s}$ period}

Considering the pair $\mathrm{D}$, the value $|\Delta m|_{\mathrm{D}}=4$ confirms that the $70.9 \mathrm{~s}$ period cannot correspond to a component of the quintuplet generated by the $\ell=2, k=1$ mode. The frequency difference between the $70.9 \mathrm{~s}$ period and the next one at $72.5 \mathrm{~s}$ is $313 \mu \mathrm{Hz}$, about 20 times the value of $\delta f_{\ell=2}$. As a consequence, the 2 periods $70.9 \mathrm{~s}$ and $72.5 \mathrm{~s}$ cannot belong to the same multiplet. This reinforces our previous conclusion that the $70.9 \mathrm{~s}$ period should be the harmonics of the $141.9 \mathrm{~s}$ mode, with $f_{70.9} \mathrm{~s}=2 \times f_{141.9 \mathrm{~s}}$.

\subsubsection{The $651.7 \mathrm{~s}$ period}

From Table 7, we could not identify the $651.7 \mathrm{~s}$ period at first sight. Taking the rotational splitting into account with $\delta f_{\ell=2} \sim$ $16 \mu \mathrm{Hz}$, we can now identify this mode with the $m=-2$ component of the $\ell=2, k=21, m=0$ mode at $633.8 \mathrm{~s}$ (Table 6), which we find at a period of $646.9 \mathrm{~s}$, with a relative error of $0.7 \%$.

\subsubsection{The $299.8 \mathrm{~s}$ and $301.4 \mathrm{~s}$ periods}

CKM identify the $299.8 \mathrm{~s}$ and $301.4 \mathrm{~s}$ periods as $\ell=1$ modes. Our analysis offers an alternative identification as $\ell=2$ modes. Our model spectrum can only match these periods with the $\ell=2, k=8$ mode with a period at $300.7 \mathrm{~s}$. The pair $299.8 \mathrm{~s}$, $301.4 \mathrm{~s}$ is equidistant from the closest computed $\ell=1$ modes at $281.2 \mathrm{~s}$ for the $k=3$ mode and at $322.0 \mathrm{~s}$ for the $k=4$ mode. This confirms that the $299.8 \mathrm{~s}$ and $301.4 \mathrm{~s}$ periods must be $\ell=2$ modes.

\subsubsection{The $212.8 \mathrm{~s}$ and $215.7 \mathrm{~s}$ periods}

We suggested in the previous section that these two periods may result from a linear combination of the mode observed at $454.6 \mathrm{~s}$ with the two interfering and undetected modes at $397.0 \mathrm{~s}$ predicted as $\ell=1$ and $\ell=2$ modes in our best-fit model. Note that it is usually postulated that in order to be identified as the linear combination of two parent mode frequencies $f_{1}$ and $f_{2}$, a frequency $f_{3}$ such that $f_{3}=f_{1} \pm f_{2}$ should be visible only when its two parents are also detected and its amplitude in the Fourier spectrum should be smaller than their respective amplitude. In the present case, the situation is different since one of the two parent modes itself results from two modes interfering at the same frequency, and could be either in a regime of destructive or constructive interference. Considering now that these two interfering modes are split by rotation, with the value of the rotational splitting derived from section 5.1, we find that the linear combination of the observed mode at $454.6 \mathrm{~s}$ with the $\ell=2$, $k=12, m=-1$ component has a period of $212.6 \mathrm{~s}$, at $0.2 \mathrm{~s}$ of the observed period $212.8 \mathrm{~s}$, and with the $m=-2$ component has a period of $213.3 \mathrm{~s}$, at $2.4 \mathrm{~s}$ of the observed period at $215.7 \mathrm{~s}$. The linear combination with the $\ell=1, k=6, m=-1$ component would explain the $212.8 \mathrm{~s}$ period (at $212.3 \mathrm{~s}$ ) almost as well but would not provide any solution for the $215.7 \mathrm{~s}$.

\subsection{Full identification of the observed modes of $\mathrm{G}$ 185-32}

Our estimate of the frequency shift for the $\ell=2$ modes allows us to complete the former preliminary identification of the periods given in Table 7 by introducing the value of the azimuthal number $m$. When no unique interpretations were possible for some periods, i.e. when two distinct modes with a different $\ell$ degree could match the observed period, we selected the solution that resulted in the least relative (and absolute) error. The value of $m$ was chosen so as to induce the most constant value of $|\delta P / P|$ within the components of a multiplet. Table 8 gives the complete identification of the modes observed in G 185-32. Taking the rotational splitting into account improves the quality of the global fit to $\sigma=1.4 \mathrm{~s}$. The average relative error decreases to $0.5 \%$ and one additional mode (the $651.7 \mathrm{~s}$ period) is successfully identified. 


\section{Discussion}

Our strategy to constrain the internal structure of the ZZ Ceti star G 185-32, based on studying its pulsation modes, resulted in obtaining a best-fit model. From this model one derives its main structural parameters: its total mass $M_{\star}, \mathrm{H}$ and He mass fractions, $T_{\text {eff }}$, radius, and luminosity. We were also able to distinguish the real modes from their linear combinations and harmonics among the observed periods. Then we deduce the degree, the order, and the azimuthal number of the real modes by using the additional information on the rotational splitting induced by the stellar rotation. In the following, we compare our results with the previous analyses by CKM and by Thomson et al. (2004).

\subsection{Determining the spherical degree of the modes}

CKM propose an identification of the $\ell$ value for 14 of the modes observed in G 185-32. They based this identification on the different variations in the modes amplitude with wavelength according to their $\ell$ value. For those periods that we identify as real modes in our analysis, we find some $\ell$ identifications that disagree with CKM. Our identifications agree with CKM for the $72.5 \mathrm{~s}, 72.9 \mathrm{~s}, 264.2 \mathrm{~s}, 266.2 \mathrm{~s}$, and $651.7 \mathrm{~s}$ modes, but they disagree for the $299.8 \mathrm{~s}, 301.4 \mathrm{~s}, 370.2 \mathrm{~s}, 454.6 \mathrm{~s}$, and $560.8 \mathrm{~s}$ periods. However, the disagreement may not be that significant. In the UV, the predicted theoretical amplitude difference between $\ell=1$ and $\ell=2$ modes is the same order of magnitude as the uncertainties on the HST data as shown by the error bars on the mode amplitudes given by CKM. As a consequence, distinguishing $\ell=1$ from $\ell=2$ modes with this method would require a much higher $\mathrm{S} / \mathrm{N}$ ratio in the UV. We also argue that some of the periods that CKM identify as real modes should instead be linear combinations.

\subsubsection{Ambiguous identifications}

A few cases where we find different identifications deserve a more detailed discussion. According to the periods of the $\ell=1$ and $\ell=2$ modes in our best-fit model, as listed in Tables 5 and 6 , there could be two possible identifications for 2 of the observed modes at $370.2 \mathrm{~s}$ and $454.6 \mathrm{~s}$. The $370.2 \mathrm{~s}$ mode could be:

- either the $\ell=2, k=11$, and $m=2$ mode, for which we find a period of $369.8 \mathrm{~s}$, which matches the observed period with a relative error $|\delta P / P|=0.1 \%$;

- or the $\ell=1, k=5$, and $m=-1$ mode, for which we find a period of $365.6 \mathrm{~s}$, which matches the observed period with a relative error $|\delta P / P|=1.2 \%$.

Our identification criterium selects the identification that minimizes the relative error, so we selected the first identification. The second solution is still satisfactory, although less pertinent, and agrees with the $\ell$ identification of CKM. However, the first identification gives a better fit with the data.

Similarly, the $454.6 \mathrm{~s}$ mode could be identified as:

- either the $\ell=2, k=14$, and $m=0$ mode, with a period of $455.2 \mathrm{~s}$ and a relative error $|\delta P / P|=0.1 \%$;

- or the $\ell=1, k=7, m=1$ mode, with a of $459.4 \mathrm{~s}$ and a relative error of $|\delta P / P|=1.1 \%$.

Once again, the first identification fits the data best, while the second one, which agrees for the $\ell$ value with CKM, cannot be absolutely rejected.
Table 8. Full identification of the observed modes in G 185-32.

\begin{tabular}{cccrccc}
\hline \hline $\begin{array}{c}\text { Observed } \\
\text { period }\end{array}$ & $\ell$ & $k$ & $m$ & $\begin{array}{c}\text { Computed } \\
\text { period }\end{array}$ & $\begin{array}{c}|\delta P| \\
(\mathrm{s})\end{array}$ & $\begin{array}{c}|\delta P / P| \\
(\%)\end{array}$ \\
\hline $72.5 \mathrm{~s}$ & 2 & 1 & 2 & $71.8 \mathrm{~s}$ & 0.7 & 1.0 \\
$72.9 \mathrm{~s}$ & 2 & 1 & -2 & $72.2 \mathrm{~s}$ & 0.7 & 1.0 \\
& & & & & & \\
$141.2 \mathrm{~s}$ & 2 & 2 & 2 & $141.5 \mathrm{~s}$ & 0.3 & 0.2 \\
$141.9 \mathrm{~s}$ & 2 & 2 & 0 & $142.1 \mathrm{~s}$ & 0.2 & 0.1 \\
$264.2 \mathrm{~s}$ & 2 & 7 & 2 & $267.0 \mathrm{~s}$ & 2.8 & 1.1 \\
$266.2 \mathrm{~s}$ & 2 & 7 & 0 & $269.3 \mathrm{~s}$ & 3.1 & 1.2 \\
$285.1 \mathrm{~s}$ & 1 & 3 & -1 & $282.0 \mathrm{~s}$ & 3.1 & 1.1 \\
$299.8 \mathrm{~s}$ & 2 & 8 & 1 & $299.3 \mathrm{~s}$ & 0.5 & 0.2 \\
$301.4 \mathrm{~s}$ & 2 & 8 & 0 & $300.7 \mathrm{~s}$ & 0.7 & 0.2 \\
$370.2 \mathrm{~s}$ & 2 & 11 & 2 & $369.8 \mathrm{~s}$ & 0.4 & 0.1 \\
& & & & & & \\
$454.6 \mathrm{~s}$ & 2 & 14 & 0 & $455.2 \mathrm{~s}$ & 0.6 & 0.1 \\
$537.6 \mathrm{~s}$ & 1 & 9 & -1 & $536.6 \mathrm{~s}$ & 1.0 & 0.2 \\
$560.8 \mathrm{~s}$ & 2 & 18 & 0 & $559.6 \mathrm{~s}$ & 1.2 & 0.2 \\
$651.7 \mathrm{~s}$ & 2 & 21 & -2 & $646.9 \mathrm{~s}$ & 4.8 & 0.7 \\
\hline
\end{tabular}

\subsubsection{The couple of periods $299.8 \mathrm{~s}$ and $301.4 \mathrm{~s}$}

We justified above the identification of the pair of periods at $299.8 \mathrm{~s}$ and $301.4 \mathrm{~s}$ as $\ell=2$ modes. It fits the observed periods with a very small relative error $(|\delta P / P|=0.2 \%)$. This disagrees with CKM, who identify those periods as $\ell=1$ modes.

\subsubsection{The couple of periods $212.8 \mathrm{~s}$ and $215.7 \mathrm{~s}$}

CKM propose an identification of the $212.8 \mathrm{~s}$ periodicity as an $\ell=1$ mode and of the $215.7 \mathrm{~s}$ one as an $\ell=2$ mode. We argue above that, by considering the beating that occurs between two modes of degree $\ell=1$ and $\ell=2$ with a similar period of $397.0 \mathrm{~s}$, we could explain the $212.8 \mathrm{~s}$ and $215.7 \mathrm{~s}$ periods as resulting from a linear combination of those modes at $397.0 \mathrm{~s}$, split by rotation, with the $454.6 \mathrm{~s}$ mode. In addition, the inability of the synthetic spectrum to reproduce those two periods strengthens this hypothesis. Provided that the $212.8 \mathrm{~s}$ and $215.7 \mathrm{~s}$ pulsations are factitious modes, the problem of their $\ell$ identification vanishes.

\subsection{The case of the peculiar $141.9 \mathrm{~s}$ mode}

Among the various singularities detected in the power spectrum of $\mathrm{G} 185-32$, the most enigmatic is the 141.9 s period whose amplitude does not vary with wavelength, contrary to what is expected from theory for $\ell=1$ or $\ell=2$ non-radial $g$-modes. Several scenarii have been proposed to explain this peculiar behaviour.

For CKM, this atypical behaviour is the signature of a nonlinear effect, and thus the $141.9 \mathrm{~s}$ period is not a true pulsation mode. This implies that the $70.9 \mathrm{~s}$ period is a real mode and, consequently, that the $141.9 \mathrm{~s}$ is its sub-harmonics. We have proposed an alternative hypothesis, considering that the $70.9 \mathrm{~s}$ period and the pair of periods at $72.5 \mathrm{~s}$ and $72.9 \mathrm{~s}$ are all genuine modes is problematic. If these three periods are real modes, either they belong to the same multiplet generated by rotation (since we have dismissed magnetic splitting as a possible explanation) or the $70.9 \mathrm{~s}$ period, one one hand, and the pair $72.5 \mathrm{~s}-72.9 \mathrm{~s}$, on the other, correspond to distinct modes with different $\ell$ degrees, either $\ell=1$ or $\ell=2$. The value we derived for the frequency shift $\left(\delta f_{\ell=2} \sim 16 \mu \mathrm{Hz}\right)$ excludes the first possibility. The frequency difference between the $70.9 \mathrm{~s}$ period and the $72.5 \mathrm{~s}$ one is by far too large to be compatible with $\delta f_{\ell=2}$. 
Since the $72.5 \mathrm{~s}$ and $72.9 \mathrm{~s}$ periods are identified as components of a $\ell=2$ mode, the result is that the $70.9 \mathrm{~s}$ period has to be an $\ell=1$ (and obviously the $k=1$ ) mode according to the second alternative, if one excludes the possibility of $\ell \geq 3$ because of the geometrical cancellation effect. This second hypothesis would imply a mass of $G$ 185-32 close to the Chandrashekar limit, since only such a very massive ZZ Ceti star could exhibit an $\ell=1, k=1$ mode with such a short period.

As already pointed out by CKM, this disagrees with the mass derived from spectroscopic observations and parallax, which is about $0.64 M_{\odot}$. As a result, the assumption that the $70.9 \mathrm{~s}$ is a real mode, and the $141.9 \mathrm{~s}$ period consequently a false one, appears very doubtful. Alternatively, we showed that identifying the $70.9 \mathrm{~s}$ with an $\ell=3$ mode would imply that it has an intrinsic amplitude at the stellar surface at least $15 \times$ larger than the $\ell=1$ mode. The mass of G $185-32$ which we derived if the $70.9 \mathrm{~s}$ is identified as either a $k=1$ or $k=2 \ell=3$ mode is outside the range of the spectroscopic mass. Furthermore, if our best-fit model does satisfactorily represent G 185-32, it does not have any $\ell=3$ mode with period close to $70.9 \mathrm{~s}$. This value falls in between the $k=1$ mode at $50.9 \mathrm{~s}$ and the $k=2$ mode at $101.2 \mathrm{~s}$.

Thompson et al. (2004) do not exclude the possibility that the $141.9 \mathrm{~s}$ period could be a genuine mode, but they do suggest that it is an $\ell=4$ non radial $g$-mode. Linear theory predicts that the amplitude for the $\ell=4 g$-modes does not vary significantly with wavelength from the UV to the visible, which is precisely what is observed for the $141.9 \mathrm{~s}$ period. However, they did not compute relevant models and their non-radial $g$-modes to check whether a suitable $\ell=4$ mode could match the observed 141.9 s pulsation, as well as the other periods observed in G 185-32. Considering the $\ell=4$ modes in the best-fit model that could best approach the $141.9 \mathrm{~s}$, we find the $k=4$ mode at $145.6 \mathrm{~s}$, at $3.7 \mathrm{~s}$ of the observed period. By taking into account the rotational splitting, which should be $\approx 18.2 \mu \mathrm{Hz}$ for the $\ell=4$ modes if it is $16 \mu \mathrm{Hz}$ for the $\ell=2$, we find that the $m=4$ component at $144.07 \mathrm{~s}$ could marginally fit the observed period within $2.17 \mathrm{~s}(1.5 \%)$. On this argument alone, we cannot dismiss the possibility that the $141.9 \mathrm{~s}$ could be an $\ell=4$ mode. However, the visibility of such modes is problematic, since the amplitude ratio of $\ell=$ 4 modes relative to $\ell=1$ modes is reduced by at least a factor of 25 (Brassard et al. 1995). It would require that the intrinsic amplitudes at the stellar surface be at least 25 times larger for the $\ell=4$ modes than for the $\ell=1$ in order to be observed with comparable amplitudes, which we consider as improbable.

We suggest another scenario to account for the peculiarity of the 141.9 s period. We found the $\ell=2, k=2, m=0$ mode in our best-fit model to have a period of $142.1 \mathrm{~s}$. We conclude that the $141.9 \mathrm{~s}$ period can be identified with this mode. However, if this identification allows us to recognize the $141.9 \mathrm{~s}$ period as a true mode, the strange behaviour of its amplitude with wavelength still needs to be understood. In computing the $\ell=3, \ell=4$ and $\ell=5$ non-radial $g$-modes in our best-fit model, whose periods are close to $140 \mathrm{~s}$, we found that the $\ell=3, k=4$ mode has a period of $139.9 \mathrm{~s}$, the $\ell=4, k=6$ a period of $145.6 \mathrm{~s}$ and the $\ell=5, k=9$ a period of $143.0 \mathrm{~s}$. One then notices that $f_{139.9 \mathrm{~s}}+f_{145.6 \mathrm{~s}} \approx 2 \times f_{141.9 \mathrm{~s}}$ with $|\Delta f|=\mid f_{141.9 \mathrm{~s}}-0.5 \times\left(f_{139.9 \mathrm{~s}}+\right.$ $\left.f_{145.6 \mathrm{~s}}\right) \mid \sim 40 \mu \mathrm{Hz}$ and that $f_{139.9 \mathrm{~s}}+f_{143.0 \mathrm{~s}} \approx 2 \times f_{141.9 \mathrm{~s}}$ with $|\Delta f|=\left|f_{141.9 \mathrm{~s}}-0.5 \times\left(f_{139.9 \mathrm{~s}}+f_{143.0 \mathrm{~s}}\right)\right| \sim 20 \mu \mathrm{Hz}$.

These relations suggest that the $\ell=2, k=2$ mode accounting for the observed $141.9 \mathrm{~s}$ period might be affected by two potential true resonances, each implying modes with 3 different $\ell$ degrees, $\ell=2,3,4$ for the first one and $\ell=2,3,5$ for the second one. The frequency differences $|\Delta f|$ are not strong concerns because they can be easily justified by the internal errors inherent to modelling and mode computation, which cannot lead to better precision on the computed periods than about $0.5 \mathrm{~s}$. This is even truer for the second potential frequency resonance for which $|\Delta f|$ is the same order of magnitude than the frequency resolution derived from the HST data set.

It is also possible that modes with degree $\ell \geq 6$ could potentially induce true resonances with the $\ell=2, k=2$ mode. However, the radial density and pressure perturbations decrease with increasing $\ell$, making the probability of interfering with the central mode $\ell=2, k=2$ of the resonant triplet less probable, or at least strongly reducing its detectability. As a consequence, we consider only the two former frequency relations as able to account for a true resonance involving the observed $(\ell=2, k=2)$ 141.9 s mode.

It is hardly surprising not to detect the two other modes involved in these resonant triplets since the visibility of the modes decreases rapidly with increasing $\ell$. For the same reason, the results of the linear combinations $\left[f_{\ell=3(139.9 \mathrm{~s})}+f_{\ell=4}(145.6 \mathrm{~s})\right]$ or $\left[f_{\ell=3(139.9 \mathrm{~s})}+f_{\ell=5(143.0 \mathrm{~s})}\right]$ would probably remain undetected if they did not combine with the $\ell=2, k=2,141.9$ s mode.

As a consequence, we suggest that the $141.9 \mathrm{~s}$ pulsation might be the result of a three-mode resonance, due to the coincidence between the frequency of a $\ell=2$ mode and the frequency of the linear combination generated by two parent modes of higher degrees, either $\ell=3$ and $\ell=4$ or $\ell=3$ and $\ell=5$. This may explain why the amplitude of the resulting pulsation does not follow the wavelength dependence predicted by linear theory for the amplitude of a single, isolated $\ell=2$ mode. One may anticipate that if such a triplet resonance occurs, the amplitude of the $\ell=2$ mode might be strongly disturbed by its interaction with the other modes. It is still necessary to prove that the wavelength dependence of the resuling pulsation agrees with the observations, which is beyond the scope of the present paper.

One may even go further than this hypothesis to also suspect that this resonant triplet is affected by the non-linear effect of frequency-lock or, at least, is in the situation of the intermediate regime discussed by Goupil et al. (1998). They showed that, if three modes of different $\ell$ degrees have frequencies very close to resonance, i.e. such as $f_{1}+f_{3} \sim 2 \times f_{2}$, then they are frequency-locked. It means that, when the frequencies are near the exact resonance, the resonant coupling resulting from their non-linear interaction forces the three frequencies to be equidistant even if they are not so in the frame of linear theory. Note that in this case the actual frequencies of the modes involved by the frequency-lock differ from the values computed for the modes in our model since our calculations rely on linear theory. However, Goupil \& Buchler (1994) have established that such a non-linear effect must comply with some selection rules. In particular, for the resonant coupling to occur, the sum of the spherical degrees of all the involved modes must be even. For the first potential resonance, the sum of the degrees is 11 $(\ell=4+\ell=3+\ell=2+\ell=2)$, so this solution is not eligible for non-linear coupling. The second potential resonance satisfies the selection criterium since it implies an even sum of degrees (12): $\ell=5+\ell=3+\ell=2+\ell=2$. It is the only possibility for a non-linear coupling to occur either in a frequency-lock or in an intermediate regime. The probability of a non-linear coupling to occur increases with decreasing frequency mismatch $|\Delta f|$. Here, $|\Delta f| \sim 20 \mu \mathrm{Hz}$ and the frequencies of the three modes are almost equidistant: $\left[f_{(141.9 \mathrm{~s})}-f_{(143.0 \mathrm{~s})}\right] \sim\left[f_{(139.9 \mathrm{~s})}-f_{(141.9 \mathrm{~s})}\right]$.

Goupil et al. (1998) have shown that the amplitude of the three modes resulting from a frequency-lock depends on the 
modes growth rate $(\kappa)$ and on some non-linear coupling coefficients. It is then possible that the triplet configuration favours the amplitude of the central component, i.e. the $141.9 \mathrm{~s}$ observed mode, and also not surprising that the amplitude of this mode does not vary with wavelength as suggested by linear theory. To settle this hypothesis unambiguously, it will be necessary to estimate the growth rate $\kappa$ of the involved modes by performing non-adiabatic calculations. According to the value derived for $\kappa$, it will be possible to check whether the triplet resonance is in the frequency-lock regime, i.e. if $\kappa \geq 2 \pi|\Delta f|$, or in the intermediate regime, if $\kappa \leq 2 \pi|\Delta f|$. This is a promising direction to explore but is also out of the scope of this paper. This suggestion could also be checked observationally since the resonant coupling induces amplitude and frequency variations, whose amplitudes and timescales depend on the status of the coupling, i.e. on whether it is in a frequency-locked or in an intermediate regime. Those predictions could be tested by long-term observations if they provide the required frequency and amplitude accuracy. The WET data are presently the only data providing the required accuracy on the frequencies (but may be not on the amplitudes) for only one season. More observations are necessary to test the validity of this assumption.

\section{Conclusions}

By comparing the observed periods with the periods of the nonradial $g$-modes of a set of models, we were able to select a model that fits best the ZZ Ceti star G 185-32 and to constrain its main physical parameters. It was then possible to identify the periods detected in its power spectrum and to distinguish the real modes from their linear combinations. We also estimated the rotational splitting, which allowed a more complete mode identification. We proposed a new hypothesis to explain the singularity of the 141.9 s mode.

Relying on the $72.5 \mathrm{~s}$ period, which we identify as the $\ell=2$, $k=1$ mode as a reference mode, the modelling process resulted in a best-fit model whose synthetic spectrum fits the observed modes of G 185-32 with an average relative error of $0.5 \%$, which we consider as a very good fit. The main structural properties of the model are: a full mass $M_{\star}=0.638( \pm 0.007) M_{\odot}$, a thick hydrogen envelope as massive as $M(\mathrm{H})=1.70( \pm 0.10) \times 10^{-4} M_{\star}$, and an effective temperature $T_{\text {eff }}=12280( \pm 80) \mathrm{K}$. These values confirm that $\mathrm{G} 185-32$ is located near the blue edge of the ZZ Ceti instability strip. Its hydrogen mass fraction is close to the one derived for the cooler ZZ Ceti star HL Tau 76: $M(\mathrm{H})=2.35 \times 10^{-4} M_{\star}(\mathrm{PVD}, 2006)$.

The presence of partial multiplets in the pulsation spectrum of $\mathrm{G}$ 185-32 allowed us to evaluate the rotational splitting for the $\ell=2$ modes, $\delta f_{\ell=2} \sim 16 \mu \mathrm{Hz}$. It implies an average rotation period of $14.5 \mathrm{hr}$, roughly 3 or 4 times faster than HL Tau 76. This rotation period is comparable to the one for G 226-29 (approximatively $9 \mathrm{~h}$ ).

The computed $\ell=1$ and $\ell=2$ modes for the best-fit model enabled us to determine the nature of some ambiguous periods, especially when they are marginally detected, as in the case of the $181.9 \mathrm{~s}$ period, for example, or when the mode satisfies a linear combination relation. Thus, our modelling finds that the $70.9 \mathrm{~s}, 148.5 \mathrm{~s}, 181.9 \mathrm{~s}, 212.8 \mathrm{~s}$, and $215.7 \mathrm{~s}$ periods are false modes.
We compared our $\ell$ identification with the one proposed by CKM. Our analysis globally agrees with CKM except in the following cases: the pair of periods 299.8 s-301.4 s, which CKM identify as an $\ell=1$ mode, are $\ell=2$ modes; the $370.2 \mathrm{~s}$ and 454.6 s periods, which CKM also identify as $\ell=1$ modes, are found to fit the observations better if they are $\ell=2$ modes. We note that $\mathrm{G} 185-32$ oscillates more often in $\ell=2$ modes, like HL Tau 76 (PVD 2006) and BPM 37093 (Kanaan et al. 2005).

Finally, we propose a new working hypothesis to explain why the amplitude of the enigmatic 141.9 s period does not vary with wavelength from the UV to the visible, contrary to what the linear theory predicts for $\ell=1$ or $\ell=2$ modes. To account for this phenomenon, CKM considered that this period was not a true mode, while Thompson et al. (2004) propose associating this period with an $\ell=4$ mode. We suggest another scenario based on calculation of the $\ell=2,3,4$, and 5 modes in our best fit model. The $141.9 \mathrm{~s}$ could result from the superposition of a real $\ell=2$ mode with a linear combination generated by two parent modes of degrees $\ell=3$ and $\ell=4$ or $\ell=3$ and $\ell=5$. As a result, the amplitude of the genuine $\ell=2$ mode would be altered by its interference with the linear combination whose frequency coincides exactly with its frequency. Accordingly, it is not surprising that the amplitude variation with wavelength of the resulting period does not comply with the theoretical predictions of an isolated, undisturbed mode. Furthermore, this resonant triplet, induced by the non-linear interaction between the three modes, may be in a frequency-lock regime or in a situation of intermediate regime. However, such a resonance is possible only between the $\ell=2,3$, and 5 modes because of selection rules. This non-linear coupling, if it occurs, could explain the strangeness of the $141.9 \mathrm{~s}$ mode, since it perturbs both the frequency and the amplitude of each mode involved in the resonant triplet.

\section{References}

Bergeron, P., Wesemael, F., Lamontagne, R., et al. 1995, ApJ, 449, 258 Bergeron, P., Fontaine, G., Billères, M., Boudreault, S., \& Green, E.M. 2004, ApJ, 600, 404

Brassard, P., Fontaine, G., Wesemael, F., \& Tassoul, M. 1992, ApJS, 81, 747

Brassard, P., Fontaine, G., \& Wesemael, F. 1995, ApJS, 96, 545

Brickhill, A. J. 1975, MNRAS, 170, 405

Castanheira, B. G., Kepler, S. O., Moskalik, P., et al. 2004, A\&A, 413, 623 (CKM)

Dolez, N., Vauclair, G., Kleinman, S. J., et al. 2006, A\&A, 446, 237

Dziembowski, W. 1977, Acta Astr., 27, 203

Goupil, M.-J., \& Buchler, J. R. 1994, A\&A, 291, 481

Goupil, M.-J., Dziembowski, W. A., \& Fontaine, G. 1998, Baltic Astronomy, 7, 21

Jones, P. W., Pesnell, W. D., Hansen, C. J., \& Kawaler, S. D. 1989, ApJ, 336, 403

Kanaan, A., Nitta, A., Winget, D. E., et al. 2005, A\&A, 432, 219

Kepler, S. O. 1984, ApJ, 278, 754

Kepler, S. O., Giovannini, O., Wood, M. A., et al. 1995, ApJ, 447, 874

Kepler, S. O., Robinson, E. L., Koester, D., et al. 2000, ApJ, 539,379

Mc Graw, J. T., Fontaine, G., Lacombe, P., et al. 1981, ApJ, 250, 349

Mukadam, A., et al. 2006, ApJ, in press [arXiv: astro-ph/0507425]

Nather, R. E., Winget, D. E., Clemens, J. C., et al. 1990, ApJ, 361, 309

Pech, D., Vauclair, G., \& Dolez, N. 2006, A\&A, 446, 223 (PVD)

Robinson, E. L., Kepler, S. O., \& Nather, R. E. 1982, ApJ, 259, 219

Robinson, E. L., Mailloux, T. M., Zhang, E., et al. 1995, ApJ, 438, 908

Schmidt, G. D., \& Smith, P. S. 1995, ApJ, 448, 305

Thomson, S. E., Clemens, J. C., van Kerkwijk, M. H., et al. 2004, ApJ, 610, 1001 